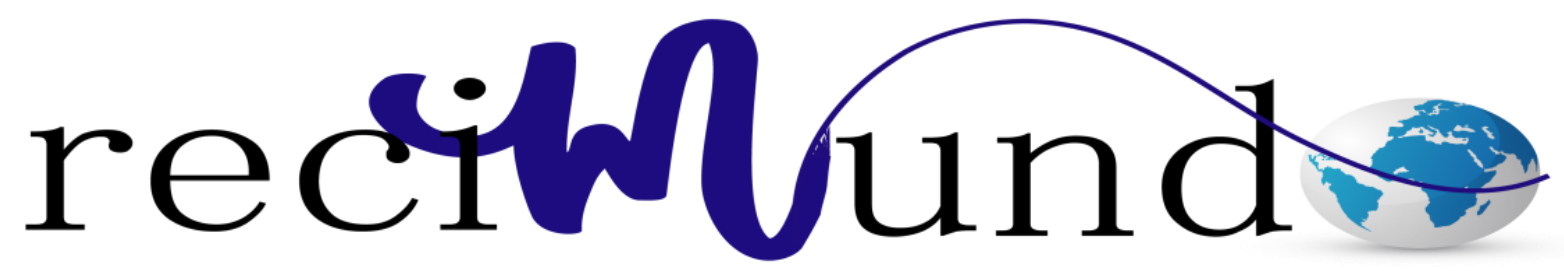

Revista Científica Mundo de la Investigación y el Conocimiento

Jorge Alexander Bucheli García a ; Wilson Oswaldo Cando Tipán ${ }^{\text {b; Santiago }}$

Patricio Muñoz González ${ }^{c}$; Oscar Jaramillo De León ${ }^{\text {d }}$

Identificación, evaluación y propuesta de medidas de control de riesgos mecánicos en la operación de una planta de trituración de agregados en la ciudad de Quito, para fabricación de mezclas de hormigón o asfalto

Identification, evaluation and proposal for mechanical risk control measures in the operation of a grinding plant of aggregates in the city of Quito, for the manufacture of concrete or asphalt mixtures

Revista Científica Mundo de la Investigación y el Conocimiento. Vol. 2 núm.3, julio, ISSN: 2588-073X, 2018, pp. 151-168

DOI: 10.26820/recimundo/2.(3).julio.2018.151-168

Editorial Saberes del Conocimiento

Recibido: 05/04/2018

Aceptado: 08/06/2018

Publicado: 30/07/2018

a. Pontificia Universidad Católica del Ecuador; jabucheli@ puce.edu.ec

b. Pontificia Universidad Católica del Ecuador; wocando@ puce.edu.ec

c. Pontificia Universidad Católica del Ecuador; smunoz491@ @uce.edu.ec

d. Pontificia Universidad Católica del Ecuador; ojaramillo602@puce.edu.ec 


\section{Identificación, evaluación y propuesta de medidas de control de riesgos mecánicos en la operación de una planta de trituración de agregados en la ciudad de Quito, para fabricación de mezclas de hormigón o asfalto}

Vol. 2, núm. 3., (2018)

Jorge Alexander Bucheli García; Wilson Oswaldo Cando Tipán; Santiago Patricio Muñoz González; Oscar Jaramillo De León

\section{RESUMEN}

Se ha identificado los riegos mecánicos en la planta trituradora, a través de un análisis individual de cada maquinaria y proceso existente en el área del proyecto: volqueta, retroexcavadora, cargadora, tolva, trituradora, bandas transportadoras, criba vibrante y molino; para su posterior evaluación.

Mediante la norma NTP - 330 (Sistema simplificado de evaluación de riesgos de accidente) se evaluaron cada uno de los riesgos identificados (probabilidad y consecuencia), en dónde la probabilidad es determinada por los factores de Riesgo y la consecuencia por la magnitud del daño, dándonos como resultado la jerarquización racional de acuerdo a la prioridad. Una vez identificado el mismo, se elaboraron las listas o cuestionarios de chequeo, y el nivel de deficiencia, utilizando la Norma NTP-324, que nos permitió identificar de manera técnica el nivel del riesgo, nivel de probabilidad y nivel de consecuencias para su posterior control.

Una vez obtenido el Nivel de Riesgo e intervención y con los resultados de la evaluación se propuso medidas de prevención, protección, control de riesgos o en su defecto minimizar sus consecuencias; de acuerdo al cumplimiento de las normativas legales existentes como medio obligatorio "Mecanismos de la Prevención de Riesgos del Trabajo. IESS, Resolución No. C.D. 513, 2010”; con la finalidad de prescindir de manera técnica los daños o pérdidas que se puedan afectar a la salud, seguridad de los empleados, el medio ambiente y la producción de la plata trituradora.

Palabras claves: Riesgo, Factor de Riesgo, Nivel de Deficiencia, Nivel de Riesgo. 


\section{Identificación, evaluación y propuesta de medidas de control de riesgos mecánicos en la operación de una planta de trituración de agregados en la ciudad de Quito, para fabricación de mezclas de hormigón o asfalto}

Vol. 2, núm. 3., (2018)

Jorge Alexander Bucheli García; Wilson Oswaldo Cando Tipán; Santiago Patricio Muñoz

González; Oscar Jaramillo De León

\section{ABSTRACT}

Mechanical risks have been identified in the crushing plant, through an individual analysis of each machinery and process existing in the project area: dump truck, backhoe loader, hopper, crusher, conveyor belts, vibrating screen and mill; for further evaluation.

Through the norm NTP - 330 (Simplified system for the evaluation of accident risks), each of the identified risks (probability and consequence) was evaluated, where the probability is determined by the risk factors and the consequence by the magnitude of the damage, giving as a result the rational hierarchy according to the priority. Once the same was identified, the checklists or questionnaires were prepared, and the level of deficiency, using the NTP-324 standard, which allowed us to identify technically the level of risk, level of probability and level of consequences for later control.

Once the Risk and Intervention Level was obtained and with the results of the evaluation, measures of prevention, protection, risk control or, in their absence, minimizing their consequences were proposed; according to compliance with existing legal regulations as mandatory means "Mechanisms for the Prevention of Occupational Risks. IESS, Resolution No. C.D. 513, 2010 "; in order to technically dispense damages or losses that may affect the health, safety of employees, the environment and the production of silver crusher.

Keywords: Risk, Risk Factor, Deficiency Level, Risk Level. 


\section{Identificación, evaluación y propuesta de medidas de control de riesgos mecánicos en la operación de una planta de trituración de agregados en la ciudad de Quito, para fabricación de mezclas de hormigón o asfalto}

Vol. 2, núm. 3., (2018)

Jorge Alexander Bucheli García; Wilson Oswaldo Cando Tipán; Santiago Patricio Muñoz González; Oscar Jaramillo De León

\section{Introducción.}

Son varios los procesos que se desarrollan para obtener la materia prima para la construcción de proyectos de ingeniería civil. Uno de los procesos básicos es la trituración de agregados, los mismos que son utilizados como materia prima en la mayoría elementos básicos para la ejecución de proyectos esenciales para el desarrollo y bienestar de nuestra comunidad.

La maquinaria es fundamental en la operación de plantas de trituración, debido a que el proceso consiste en efectuarlo de manera mecánica, por la dureza de los elementos. En los procesos de trituración de agregados, los riesgos mecánicos son los más comunes, debido al tipo de maquinaria utilizada; los mismos que pueden llegar a afectar de manera negativa sobre la salud de los trabajadores produciendo: cortes, enganches, abrasiones, punciones, contusiones, proyecciones, atrapamiento, aplastamiento, cizallamiento, entre otros. Los riesgos mecánicos producen bajas por enfermedad e incapacidad laboral incrementando los costos de producción de las empresas.

Las fuentes más comunes de riesgos mecánicos son las partes en movimiento no protegidas, cualquier componente expuesto, puntos de corte, cualquier componente de máquina que se mueve con rapidez y con la energía necesaria para golpear, aplastar o cualquier otra manera de producir daños al trabajador y los lugares de operación.

El Objetivo de la presente investigación es identificar, Evaluar y Proponer medidas de control de riesgos mecánicos en la operación de una planta de trituración de agregados, para fabricación de mezclas de hormigón o asfalto. 
Identificación, evaluación y propuesta de medidas de control de riesgos mecánicos en la operación de una planta de trituración de agregados en la ciudad de Quito, para fabricación de mezclas de hormigón o asfalto

Vol. 2, núm. 3., (2018)

Jorge Alexander Bucheli García; Wilson Oswaldo Cando Tipán; Santiago Patricio Muñoz

González; Oscar Jaramillo De León

\section{Metodología.}

METODO- NTP 330

El proceso de evaluación se identifica los puestos o lugares de trabajo de trabajo y la maquinaria que se utiliza en cada uno de los mismos.

En la maquinaria y equipo de trituración se determinó los siguientes riesgos:

- Caída de personas a distinto nivel.

- Caída de material o entierro por manipulación y desplome.

- Pisadas del personal sobre objetos.

- Golpes contra elementos móviles, inmóviles objetos y herramientas.

- Atrapamiento por vuelco de maquinaria.

- Atropellos, golpes y choques con o contra vehículos.

- Proyección de fragmentos y partículas.

- Atrapamiento por objetos o material.

En el proceso de evaluación nos indica que debemos obtener ciertos valores para continuar con la evaluación, estos valores corresponden a los siguientes niveles, como lo dicta la norma, para cada uno de los riesgos en análisis.

- Nivel de Deficiencia. 


\section{Identificación, evaluación y propuesta de medidas de control de riesgos mecánicos en la operación de una planta de trituración de agregados en la ciudad de Quito, para fabricación de mezclas de hormigón o asfalto}

Vol. 2, núm. 3., (2018)

Jorge Alexander Bucheli García; Wilson Oswaldo Cando Tipán; Santiago Patricio Muñoz González; Oscar Jaramillo De León

- Nivel de Exposición.

- Nivel de Probabilidad.

- Nivel de Consecuencias.

- Nivel de Riesgo.

- Nivel de Intervención.

(INSHT, Bestratén Belloví, \& Pareja Malagón, NTP-330, 1993)

Nivel de Deficiencia $(N d)$.

El nivel de deficiencia es determinado por el riesgo considerado y la relación que puede tener con la probabilidad del accidente. (INSHT, Bestratén Belloví, \& Pareja Malagón, NTP$330,1993)$

Para la determinación de factores de riesgo debemos realizar listas de chequeo por cada riesgo mecánico analizado, las cuales deben tener factores de riesgo y por cada factor de iesgo un nivel de deficiencia. (INSHT, Bestratén Belloví, \& Pareja Malagón, NTP-330, 1993) 
Identificación, evaluación y propuesta de medidas de control de riesgos mecánicos en la operación de una planta de trituración de agregados en la ciudad de Quito, para fabricación de mezclas de hormigón o asfalto

Vol. 2, núm. 3., (2018)

Jorge Alexander Bucheli García; Wilson Oswaldo Cando Tipán; Santiago Patricio Muñoz

González; Oscar Jaramillo De León

\begin{tabular}{|c|c|c|c|c|c|c|}
\hline \multicolumn{7}{|c|}{ MAQUINARIA } \\
\hline \multicolumn{2}{|c|}{ RIES GO A ANALIZAR: } & \multicolumn{3}{|c|}{ ERSONA S A DISTINTO NIVEL } & & \\
\hline \multicolumn{2}{|c|}{ PUES TO DE TRABAJO ANALIZADO: } & \multicolumn{5}{|c|}{ OPERADOR } \\
\hline $\mathbf{N}^{\circ}$ & FACTOR DE RIESGO & ND & $\mathrm{N} / \mathrm{D}(\mathrm{OBT})$ & $\underline{\mathrm{SI}}$ & $\mathrm{NO}$ & $\mathrm{N} / \mathrm{A}$ \\
\hline \multicolumn{7}{|c|}{$\overline{\text { AGENTES MATERIALES }}$} \\
\hline 1 & $\begin{array}{l}\text { ¿Se realiza una limpieza previa } \\
\text { del mecanismo de acceso a la } \\
\text { volqueta? }\end{array}$ & 6 & & & & \\
\hline 2 & $\begin{array}{l}\text { ¿Se utiliza un medio de } \\
\text { transporte adecuado para el } \\
\text { transporte de personas? }\end{array}$ & 6 & & & & \\
\hline 3 & $\begin{array}{l}\text { ¿El operador supervisa que las } \\
\text { personas no deben subir o bajar } \\
\text { de la maquinaria? }\end{array}$ & 2 & & & & \\
\hline 4 & $\begin{array}{l}\text { ¿La forma correcta de subir o } \\
\text { bajar de la maquinaria es de } \\
\text { forma frontal utilizando las } \\
\text { gradas y barandillas, sin saltar? }\end{array}$ & 3 & & & & \\
\hline 5 & $\begin{array}{l}\text { ¿Se realiza una capacitación } \\
\text { para el uso correcto de los } \\
\text { accesos a la maquinaria? }\end{array}$ & 3 & & & & \\
\hline & & ND & & & & \\
\hline
\end{tabular}

\section{Gráfico 1: Lista de Chequeo}

Fuente: La autora

Mediante la investigación de los reglamentos, normativas y decretos nacionales e internacionales se identifica los factores de riesgo (circunstancias o situaciones), que podrían aumentar la materialización de los mismos. De acuerdo a las funciones en cada área de trabajo, se tiene diferentes factores de riesgo, para cada uno de estos se realiza la sustentación legal correspondiente. Todos los factores de riesgos tienen un nivel de deficiencia que va en el rango de 1-10.

Las preguntas de la lista de chequeo se formulan de tal forma que cuando las respuestas son negativas, se demuestre que el nivel de deficiencia aumenta. 
Identificación, evaluación y propuesta de medidas de control de riesgos mecánicos en la operación de una planta de trituración de agregados en la ciudad de Quito, para fabricación de mezclas de hormigón o asfalto

Vol. 2, núm. 3., (2018)

Jorge Alexander Bucheli García; Wilson Oswaldo Cando Tipán; Santiago Patricio Muñoz González; Oscar Jaramillo De León

Utilizando una metodología para cada uno de los factores de riesgo se determina el nivel de deficiencia ponderada. Para la obtención de los resultados de la evaluación, se pondera el nivel de deficiencia, de tal manera, que si todas las respuestas de la lista de chequeo son negativas sumen un total de 10 puntos.

\begin{tabular}{|c|c|c|c|c|c|c|c|}
\hline \multicolumn{8}{|c|}{ VOLQUETA } \\
\hline \multicolumn{2}{|c|}{ RIESGO AANALUZAR: } & CAIDA DE PI & ERSONAS & A DISTIN & NTO & & \\
\hline \multicolumn{2}{|c|}{ PUESTO DE TRABAJO ANALZZADO: } & \multicolumn{6}{|c|}{ OPERADOR } \\
\hline$N^{\circ}$ & \multicolumn{2}{|c|}{ FACTOR DE RIESGO } & ND & \multicolumn{2}{|c|}{$\underline{\mathrm{N} D(\mathrm{OBT})} \underline{\mathrm{SI}}$} & NO & $\mathrm{N} / \mathrm{A}$ \\
\hline \multicolumn{8}{|c|}{ AGENTES MATERIALES } \\
\hline 1 & \multicolumn{2}{|c|}{$\begin{array}{l}\text { ¿ Se realiza una limpieza previa del mecanismo de } \\
\text { acceso a la volqueta? }\end{array}$} & 6 & 0,00 & $\mathrm{X}$ & & \\
\hline 2 & \multicolumn{2}{|c|}{$\begin{array}{l}\text { ¿ Se utiliza un medio de transporte adecuado para el } \\
\text { transporte de personas? }\end{array}$} & 6 & 3,00 & & $\mathrm{x}$ & \\
\hline 3 & \multicolumn{2}{|c|}{$\begin{array}{l}\text { ¿El operador supervisa que las personas no deben } \\
\text { subir o bajar de la maquinaria? }\end{array}$} & 2 & 0,00 & $\mathrm{X}$ & & \\
\hline 4 & \multicolumn{2}{|c|}{$\begin{array}{l}\text { ¿ La forma correcta de subir o bajar de la maquinaria } \\
\text { es de forma frontal utilizando las gradas y barandillas, } \\
\text { sin saltar? }\end{array}$} & 3 & 1,50 & & $\mathrm{X}$ & \\
\hline 5 & \multicolumn{2}{|c|}{$\begin{array}{l}\text { ¿Se realiza una capacitación para el uso correcto de } \\
\text { los accesos a la maquinaria? }\end{array}$} & 3 & & & & \\
\hline & & & ND & \multicolumn{3}{|c|}{$\frac{0,00 \mid \Lambda}{4,50}$} & \\
\hline
\end{tabular}

\section{Gráfico 2: Lista de Chequeo evaluada.}

Fuente: La autora

La norma NTP- 324 establece 4 grupos de factores de riesgos, el presente plan de disertación es enfocado a los riesgos mecánicos por lo cual está enfocado directamente al grupo de Agentes Materiales. (INSHT \& Piqué Ardanuy, NTP-324, 1993)

El Nivel de Exposición es determinado de forma objetiva, de acuerdo al tiempo de permanencia del operador en el lugar de trabajo, el mismo que está dado en un rango de 1-4, 


\section{Identificación, evaluación y propuesta de medidas de control de riesgos} mecánicos en la operación de una planta de trituración de agregados en la ciudad de Quito, para fabricación de mezclas de hormigón o asfalto

Vol. 2, núm. 3., (2018)

Jorge Alexander Bucheli García; Wilson Oswaldo Cando Tipán; Santiago Patricio Muñoz

González; Oscar Jaramillo De León

entendiéndose como 1 a la exposición esporádica y como 4 a una exposición frecuente. En este caso para el riesgo en análisis tomamos un valor de 2, el cual significa una Exposición Ocasional

Con estos dos datos se realiza el cálculo del Nivel de Probabilidad, resultado del producto del Nivel de Deficiencia y el Nivel de Exposición. El Nivel de Exposición puede variar de 2-40, entendiéndose como su valor máximo el Nivel de Probabilidad Muy Alta y como su valor mínimo el Nivel de Probabilidad Baja.

$$
\mathrm{NP}=\mathrm{ND} X \mathrm{NE}
$$

ND: Nivel de Deficiencia.

NE: Nivel de Exposición.

El Nivel de Consecuencia es determinado mediante daños personales y materiales, tomando en cuenta que los daños personales van a ser de mayor importancia que los daños materiales; el rango va de 10-100, siendo 10 igual a Leve y 100 Muy Catastrófico. El nivel de consecuencia de este riesgo fue de 10, de acuerdo a las estimaciones de accidentes.

El Nivel de Riesgo se puede traducir en el nivel de intervención, esta medida indica la orientación del nivel de injerencia que podemos tener en los riesgos y sirve para proponer medidas de control de los mismos. Los resultados de la evaluación se muestran en la siguiente tabla.

\section{Resultados.}


Identificación, evaluación y propuesta de medidas de control de riesgos mecánicos en la operación de una planta de trituración de agregados en la ciudad de Quito, para fabricación de mezclas de hormigón o asfalto

Vol. 2, núm. 3., (2018)

Jorge Alexander Bucheli García; Wilson Oswaldo Cando Tipán; Santiago Patricio Muñoz González; Oscar Jaramillo De León

\begin{tabular}{|c|c|c|c|c|c|c|c|c|}
\hline \multicolumn{9}{|c|}{ RESUMEN DE LA EVALUACION DE LOS RIESGOS DE UNA PLANTA DE TRITURACION DE AGREGADOS } \\
\hline $\begin{array}{l}\text { MAQUINARIA Y } \\
\text { EQUIPO }\end{array}$ & 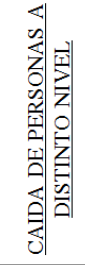 & 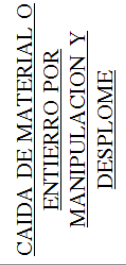 & $\begin{array}{lll} & \\
& \\
3\end{array}$ & 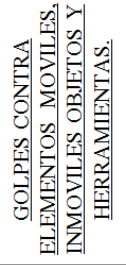 & 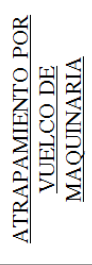 & 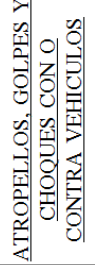 & 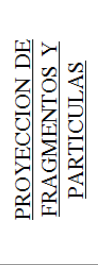 & 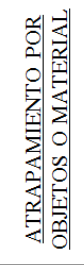 \\
\hline VOLQUETA & 90,00 & 535,71 & 88,89 & & 457,14 & & 90,91 & 67,80 \\
\hline RETROEXCAVADORA & 120,00 & 214,29 & 133,33 & 553,19 & 492,86 & 844,44 & 90,91 & 145,76 \\
\hline CARGADORA & 240,00 & 200,00 & 133,33 & 1276,60 & 407,14 & 888,89 & 136,36 & 177,97 \\
\hline TOLVA & 1462,50 & 592,59 & 88,89 & 239,36 & 0,00 & 0,00 & 60,61 & 168,83 \\
\hline TRITURADORA & 609,38 & 118,52 & 133,33 & 239,36 & 0,00 & 0,00 & & 1974,03 \\
\hline $\begin{array}{c}\text { BANDAS } \\
\text { TRANSPORTADORAS }\end{array}$ & 375,00 & 268,29 & 52,17 & 950,94 & 0,00 & 0,00 & 375,00 & 2426,23 \\
\hline CRIBA VIBRANTE & & 296,30 & 133,33 & 159,57 & 0,00 & 0,00 & 1454,55 & 340,91 \\
\hline MOLIENDA & 343,75 & 59,26 & 44,44 & 223,40 & 0,00 & 0,00 & 113,64 & 654,55 \\
\hline \multicolumn{6}{|c|}{ SITUACION CRITICA } & & & \\
\hline & & & \multicolumn{3}{|c|}{ CORREGIR Y ADOPTAR MEDIDAS } & & & \\
\hline & & & \multicolumn{3}{|c|}{ MEJORAR SI ES POSIBLE } & & & \\
\hline
\end{tabular}

Tabla 1: Resultados totales de la evaluación

PROPUESTA DE MEDIDAS DE CONTROL

o VOLQUETA

- Dotar de vainas y envolturas protectoras, para las herramientas cortopunzantes.

- Distribuir correctamente las áreas de trabajo y señalizar cada una de las mismas.

- Señalizar todas las áreas de circulación y distribuir las áreas de trabajo.

- Señalizar temporalmente el radio de acción de la volqueta mientras esta parada.

- Realizar un instructivo en el que conste las máximas velocidades de circulación dentro de la planta,

- Capacitar al personal acerca de la importancia de la señalización. 
Identificación, evaluación y propuesta de medidas de control de riesgos mecánicos en la operación de una planta de trituración de agregados en la ciudad de Quito, para fabricación de mezclas de hormigón o asfalto

Vol. 2, núm. 3., (2018)

Jorge Alexander Bucheli García; Wilson Oswaldo Cando Tipán; Santiago Patricio Muñoz

González; Oscar Jaramillo De León

- Proveer de una persona capacitada en señalización, para dar indicaciones al operador cuando la maquinaria se encuentre en movimiento

o RETROEXCAVADORA.

- Señalizar todas las áreas de circulación y distribuir las áreas de trabajo.

- Señalizar temporalmente el radio de acción de la volqueta mientras esta parada.

- Realizar un instructivo en el que conste las máximas velocidades de circulación dentro de la planta.

- Capacitar al personal acerca de la importancia de la señalización.

- Revisar que los retrovisores estén en buenas condiciones y en caso de que faltase, reemplazarlos de manera inmediata.

- Proveer de una persona capacitada en señalización, para dar indicaciones al operador cuando la maquinaria se encuentre en movimiento.

o CARGADORA.

- Dotar de vainas y envolturas protectoras, para las herramientas cortopunzantes.

- Distribuir correctamente las áreas de trabajo y señalizar cada una de las mismas.

- Capacitar a los operadores acerca de los elementos móviles de la maquinaria y su importancia.

- Señalizar todas las áreas de circulación y distribuir las áreas de trabajo.

- Señalizar temporalmente el radio de acción de la volqueta mientras esta parada. 


\section{Identificación, evaluación y propuesta de medidas de control de riesgos mecánicos en la operación de una planta de trituración de agregados en la ciudad de Quito, para fabricación de mezclas de hormigón o asfalto}

Vol. 2, núm. 3., (2018)

Jorge Alexander Bucheli García; Wilson Oswaldo Cando Tipán; Santiago Patricio Muñoz González; Oscar Jaramillo De León

- Realizar un instructivo en el que conste las máximas velocidades de circulación dentro de la planta.

- Capacitar al personal acerca de la importancia de la señalización.

- Proveer de una persona capacitada en señalización, para dar indicaciones al operador cuando la maquinaria se encuentre en movimiento.

o TOLVA.

- Se debe acceder por medio de escaleras de escaleras que tengan un ancho de $60 \mathrm{~cm}$, altura similar de todos los escalones, material antideslizante.

- Las pasarelas deben estar limpias y sin obstáculos, dotadas de barandillas.

- En escaleras con un ángulo mayor de $60^{\circ}$ se debe poner aros anticaidas.

- Las barandillas deben estar normalizadas.

- Deben estar completas y en buen estado.

- Se debe acceder por medio de escaleras de escaleras que tengan un ancho de $60 \mathrm{~cm}$, altura similar de todos los escalones, material antideslizante.

- Las pasarelas deben estar limpias y sin obstáculos, dotadas de barandillas.

- En escaleras con un ángulo mayor de $60^{\circ}$ se debe poner aros anticaidas.

- Las barandillas deben estar normalizadas.

- Deben estar completas y en buen estado. 
Identificación, evaluación y propuesta de medidas de control de riesgos mecánicos en la operación de una planta de trituración de agregados en la ciudad de Quito, para fabricación de mezclas de hormigón o asfalto

Vol. 2, núm. 3., (2018)

Jorge Alexander Bucheli García; Wilson Oswaldo Cando Tipán; Santiago Patricio Muñoz

González; Oscar Jaramillo De León

- Delimitar el área donde haya más peligro de proyección de partículas.

- Revisar que las guardas y pantallas protectoras se encuentren se encuentren en perfecto estado, mediante un plan de mantenimiento semestral.

- Implementar un sistema de riego para evitar la formación de polvo

- Controlar que las guardas y rejillas estén bien ajustadas.

- Realizar un mantenimiento preventivo al sistema de desatasco.

- Proporcionar de resguardos en las zonas donde existe mayor riesgo y exposición a los elementos móviles.

- Gestionar permisos de trabajo de acuerdo al tipo de trabajo a realizarse.

- Los problemas de atasco o imprevisto se lo realizara con herramienta sin la intervención directa de los operadores.

- Dotar de vainas y envolturas protectoras, para las herramientas cortopunzantes.

- Revisar periódicamente que los elementos móviles estén en buen estado y ajustados.

- Capacitar al personal sobre el daño que puede causar rodillo alisador (elemento móvil).

- Controlar que las guardas y rejillas estén bien ajustadas.

- Proporcionar de resguardos en las zonas donde existe mayor riesgo y exposición a los elementos móviles.

- Gestionar permisos de trabajo de acuerdo al tipo de trabajo a realizarse. 


\section{Identificación, evaluación y propuesta de medidas de control de riesgos mecánicos en la operación de una planta de trituración de agregados en la ciudad de Quito, para fabricación de mezclas de hormigón o asfalto}

Vol. 2, núm. 3., (2018)

Jorge Alexander Bucheli García; Wilson Oswaldo Cando Tipán; Santiago Patricio Muñoz González; Oscar Jaramillo De León

- Los problemas de atasco o imprevisto se lo realizara con herramienta sin la intervención directa de los operadores.

- Instruir al personal acerca de la importancia de las rejillas y resguardos de protección.

o CRIBA VIBRANTE.

- Se debe acceder por medio de escaleras de escaleras que tengan un ancho de $60 \mathrm{~cm}$, altura similar de todos los escalones, material antideslizante.

- Las pasarelas deben estar limpias y sin obstáculos, dotadas de barandillas. Deben estar completas y en buen estado

- En escaleras con un ángulo mayor de $60^{\circ}$ se debe poner aros anticaidas.

- Delimitar el área donde haya más peligro de proyección de partículas.

- Entregar el equipo de protección personal adecuado para mitigar los efectos de la proyección de partículas.

- Planificar un mantenimiento preventivo de los elementos de resguardos y pantallas protectoras.

o MOLIENDA.

- Realizar un mantenimiento preventivo del sistema de desatasco.

- Gestionar permisos de trabajo de acuerdo al tipo de trabajo a realizarse.

- Los problemas de atasco o imprevisto se lo realizara con herramienta sin la intervención directa de los operadores. 
Identificación, evaluación y propuesta de medidas de control de riesgos mecánicos en la operación de una planta de trituración de agregados en la ciudad de Quito, para fabricación de mezclas de hormigón o asfalto

Vol. 2, núm. 3., (2018)

Jorge Alexander Bucheli García; Wilson Oswaldo Cando Tipán; Santiago Patricio Muñoz

González; Oscar Jaramillo De León

- Instruir al personal acerca de la importancia de las rejillas y resguardos de protección.

\section{Conclusiones.}

Del proyecto de tesis efectuado, se puede indicar las siguientes conclusiones:

- Los riesgos mecánicos evaluados en la operación de la planta de trituración de agregados, difieren tanto en la maquinaria móvil como en la maquinaria permanente. Estos riesgos identificados corresponden a: Caída de Personas a Distinto Nivel, Caída de Material o Entierro por Manipulación y Desplome, Pisadas del Personal Sobre Objetos, Golpes Contra Elementos Móviles, Inmóviles Objetos y Herramientas, Atrapamiento por Vuelco de Maquinaria, Atropellos, Golpes y Choques Con o Contra Vehículos, Proyección de Fragmentos y Partículas, Atrapamiento por Objetos o Material; en el proceso de abastecimiento de material (Volqueta, Retroexcavadora, Cargadora).

- En el proceso de trituración primaria y trituración secundaria, se identificaron los siguientes riesgos: Caída de Personas a Distinto Nivel, Caída de Material o Entierro por Manipulación y Desplome, Pisadas del Personal Sobre Objetos, Golpes Contra Elementos Móviles, Inmóviles Objetos y Herramientas, Proyección de Fragmentos y Partículas, Atrapamiento por Objetos o Material. Además, estos riesgos están asociados a los lugares 
Identificación, evaluación y propuesta de medidas de control de riesgos mecánicos en la operación de una planta de trituración de agregados en la ciudad de Quito, para fabricación de mezclas de hormigón o asfalto

Vol. 2, núm. 3., (2018)

Jorge Alexander Bucheli García; Wilson Oswaldo Cando Tipán; Santiago Patricio Muñoz González; Oscar Jaramillo De León

de trabajo ubicados en la Tolva, Trituradora, Bandas Transportadoras, Criba Vibrante y la Molienda.

- Los riesgos mecánicos fueron evaluados en base a la norma española NTP-330 (Sistema simplificado de evaluación de riesgos de accidente). De acuerdo a esta norma, se efectuó las listas de chequeo por cada riesgo identificado, con sus respectivos factores de riesgo.

- De acuerdo a la norma española NTP-324 (Cuestionario de chequeo para el control de riesgos de accidente), se designó un nivel de deficiencia para cada factor de riesgo que existe en el proceso de trituración de agregados; para que con esta evaluación efectuada y de una manera orientativa y consecuente se pueda proponer de manera pertinente la implementación de medidas preventivas.

- De los resultados de la evaluación de riesgos mecánicos que se muestra en la tabla $\mathrm{N}^{\circ} 1$, basados en el método INSHT España, NTP-330(Sistema simplificado de evaluación de riesgos de accidente), los altos niveles de riesgo por intervención de categoría I, corresponden a: Caídas de Personas a distinto nivel; golpes contra elementos móviles, inmóviles objetos y herramientas; atropellos, golpes, choques con o contra vehículos; proyección de fragmentos y partículas; y atrapamiento por objetos o material que van con valoración desde 606,06 hasta 2426.23; indicándonos que estos riesgos son críticos y que se necesita una corrección urgente en el tipo de maquinaria a los cuales representa; a excepción de la máquina cargadora que tiene un nivel de riesgo II y III.

- De los resultados de la evaluación de riesgos mecánicos que se muestra en la tabla $\mathrm{N}^{\circ} 1$, basados en el método INSHT España, NTP-330(Sistema simplificado de evaluación de 
Identificación, evaluación y propuesta de medidas de control de riesgos mecánicos en la operación de una planta de trituración de agregados en la ciudad de Quito, para fabricación de mezclas de hormigón o asfalto

Vol. 2, núm. 3., (2018)

Jorge Alexander Bucheli García; Wilson Oswaldo Cando Tipán; Santiago Patricio Muñoz

González; Oscar Jaramillo De León

riesgos de accidente), los niveles bajos de riesgo por intervención de categoría II y III, corresponden a: Caída de Personas a Distinto Nivel; Caída de Material o Entierro por Manipulación y Desplome; Pisadas del Personal Sobre Objetos; Golpes Contra Elementos Móviles; Inmóviles Objetos y Herramientas, Atrapamiento por Vuelco de Maquinaria; Atropellos, Golpes y Choques Con o Contra Vehículos; Proyección de Fragmentos y Partículas; Atrapamiento por Objetos o Material; en los que se debe mejorar su nivel de deficiencia a través de la adopción de medidas de control para toda la maquinaria que intervienen en este proceso.

- Las medidas de control y mitigación de riesgos, fueron desarrolladas de acuerdo al nivel de intervención, enfocadas en cada uno de los riesgos evaluados por la maquinaria del proceso de una planta trituradora; y están orientados a la capacitación, señalización, delimitación y utilización de equipos de protección personal, con la finalidad de que con estas medidas propuestas se pueda corregir, eliminar, controlar o a su vez disminuirlos y así evitar accidentes laborales.

\section{Bibliografía.}

ANEFA. (2004). Prevencion de riesgos laborales en plantas de tratamiento de aridos. Madrid: ANEFA.

Henao Robledo, F. (2013). Riesgos en la construccion. Bogotá, Colombia: Ecoe ediciones.

Henao Robledo, F. (2014). Riesgo eléctricos y mecánicos. Bogotá, Colombia: Ecoe Ediciones.

IESS. (2004). Instrumento Andino de Seguridad y Salud en el Trabajo. Quito: Decisión No. 584.

IESS. (2010). Reglamento del seguro general de riesgos del trabajo. Quito: Resolución No. C.D. 513. 


\section{Identificación, evaluación y propuesta de medidas de control de riesgos mecánicos en la operación de una planta de trituración de agregados en la ciudad de Quito, para fabricación de mezclas de hormigón o asfalto}

Vol. 2, núm. 3., (2018)

Jorge Alexander Bucheli García; Wilson Oswaldo Cando Tipán; Santiago Patricio Muñoz González; Oscar Jaramillo De León

INSHT, \& Piqué Ardanuy, T. (1993). NTP-324. Obtenido de INSHT: http://www.insht.es/InshtWeb/Contenidos/Documentacion/FichasTecnicas/NTP/Ficheros 1301a400/ntp_324.pdf

INSHT, Bestratén Belloví, M., \& Pareja Malagón, F. (1993). NTP-330. Obtenido de INSHT: http://www.insht.es/InshtWeb/Contenidos/Documentacion/FichasTecnicas/NTP/Ficheros 1301a400/ntp_330.pdf

MINISTERIO DE TRABAJO. (1994). REGLAMENTO DE SEGURIDAD Y SALUD DE LOS TRABAJADORES Y MEJORAMIENTO DEL MEDIO AMBIENTE DE TRABAJO. Obtenido http://www.industrias.ec/archivos/CIG/file/SEGURIDAD/REGLAM_SEGUR_SALUD_ AMBIENTE_TRABAJO.pdf

Ministerio de Trabajo y Empleo. (10 de Enero de 2008). Registro Oficial. Obtenido de $\mathrm{N}^{\mathrm{o}} 249$ : file:///C:/Users/Luis/Downloads/REGISTRO\%20OFICIAL\%20249.pdf

Organizacion Internacional del Trabajo Ginebra. (1992). Seguridad y salud en la Construcción. Obtenido de OIT: http://www.ilo.org/wcmsp5/groups/public/---ed_protect/---protrav/--safework/documents/instructionalmaterial/wcms_218620.pdf

SEOPAN. (2010). Fichas Informativas para la Prevención de riesgos laborales en la utilización de equipos. Madrid: SEOPAN. 